\title{
Retinal Involvement in a Patient with Cerebral Manifestation of Chronic Graft-Versus-Host-Disease
}

\author{
Christiane Blecha ${ }^{a} \quad K^{2}$ lemens Angstwurm ${ }^{b}$ Daniel Wolffc Ernst Holler ${ }^{c}$ Horst Helbig ${ }^{a}$ \\ Jochen Grassinger $^{c}$ Janine Rennert ${ }^{d}$ Ingo Kleiter $^{\mathrm{e}}$ Elisabeth Huber ${ }^{f}$ Christoph Schmid ${ }^{g}$ \\ Tina Dietrich-Ntoukas ${ }^{a, h}$
}

${ }^{a}$ Department of Ophthalmology, University Medical Center Regensburg, Germany;

${ }^{b}$ Department of Neurology, University Medical Center Regensburg, Germany;

${ }^{c}$ Department of Internal Medicine III, University Medical Center Regensburg, Germany;

dInstitute of Radiology, University Medical Center Regensburg, Germany;

e Department of Neurology, St. Josef-Hospital, Ruhr-University Bochum, Germany;

${ }^{f}$ Department of Pathology, University Medical Center Regensburg, Germany;

${ }^{9}$ Department of Internal Medicine II, Klinikum Augsburg, Germany;

${ }^{\text {h}}$ Department of Ophthalmology, Charité University Medicine Berlin, Germany

\section{Established Facts}

- High likelihood of a chronic graft-versus-host disease (cGvHD) manifestation in the central nervous system (in accordance to consensus criteria) since: (1) neurological symptoms correlated closely with the intensity of immunosuppression (IS); (2) worsening of symptoms occurred during taper of IS; (3) other causes were excluded; and (4) neurological symptoms corresponded with magnetic resonance imaging abnormalities and abnormal cerebrospinal fluid.

\section{Novel Insights}

- First case of putative retinal involvement in a patient with cerebral manifestation of cGvHD.

Keywords

Chronic graft-versus-host-disease · Hematology

\section{Summary}

Background: We report a 35-year-old female patient with cerebral manifestations of chronic graft-versus-host disease (cGvHD) and putative retinal involvement after allogeneic peripheral blood stem cell transplantation (alloHSCT). Patient and Methods: The patient experienced recurrent episodes of fever and encephalitic signs 7 months after alloHSCT during taper of immunosuppression (IS). Results: Cerebral magnetic resonance imaging (MRI) showed non-gadolinium-enhancing confluent periventricular lesions and cerebrospinal fluid inflammation. After exclusion of infectious causes, treatment with steroids and antiepileptics improved cognitive deficits.

Christiane Blecha and Klemens Angstwurm contributed equally to this manuscript.
Steroid reduction provoked a relapse responding to IS. 2 years later, she complained of right-sided blurred vision and floaters; both eyes showed whitish circumscribed retinal infiltrations, cellular infiltration of the vitreous and mild bilateral optic disc edema. Oncological and neurological work-up ruled out infectious diseases and other GvHD manifestations. Symptoms and signs resolved under continued systemic IS, leaving pigmented retinal scars. After IS withdrawal, classical cutaneous cGvHD developed, resolving on systemic IS. 94 months after transplantation, she is doing well. Conclusion: To our knowledge, this is the first observation of retinal involvement of cerebral cGvHD manifestations with retinal infiltrations documented in the absence of other causes and in parallel to periventricular lesions in cerebral MRI. Based on bone marrow histology, we discuss a small vessel pathophysiology of cGvHD.

(c) 2015 S. Karger GmbH, Freiburg 


\section{Introduction}

Graft-versus-host disease (GvHD) after allogeneic peripheral blood stem cell transplantation (alloHSCT) can affect multiple organs including skin, genital and oral mucosa, lungs, liver, muscles, the skeletal system, and the peripheral and central nervous system. Ocular involvement in chronic GvHD (cGvHD) is mainly associated with ocular surface disorders [1-3]. In rare cases, the vitreous and choroids are affected in acute GvHD [4]. Retinal manifestation of $c \mathrm{GvHD}$ has not been described.

\section{Case Report}

Due to a myelodysplastic syndrome RAEB I (refractory anemia with excess blasts type 1), a 35-year old female patient received an alloHSCT from a human leukocyte antigen-matched (10/10) unrelated donor after toxicity-reduced conditioning with fludarabin, BCNU (carmustine) and melphalan, and GvHD prophylaxis with ATG (anti-thymocyte globulin), tacrolimus and MMF (mycophenolate mofetil) [5]. On day 20 she developed a GvHD with cutaneous (grade 2) and upper gastrointestinal (grade 1) involvement that responded to steroids and etanercept. Over the next months, immunosuppression (IS) was reduced as planned. On day 159 (after withdrawal of steroids, during taper of tacrolimus), she experienced repeated left-sided hemihypesthesia for some minutes and presented for neurological assessment. Clinical examination was normal, but for symmetric postural hand tremor. Cerebral MRI (magnetic resonance imaging; Siemens Symphony TIM, 1.5T, Germany) was unremarkable, but electroencephalography (EEG) revealed diffuse slowing. Focal epileptic seizures were discussed. On day 208 after alloHSCT, IS was terminated. During the following 2 months, she developed intermittent fever and progressive personality change. After a tonic-clonic seizure, she remained disorientated with psychomotor slowing and reduced awareness, spontaneous nystagmus to the left, generalized hypersensitivity to touch and hypesthesia in all sacral dermatomes on both sides. Meningeal, and other spinal and cauda-equine signs were normal. There were no signs of GvHD of any other organ and no signs of transplantation-associated microangiopathy. EEG revealed diffuse and parietal left regional slowing, and cerebral MRI remained unremarkable. Analysis of the cerebrospinal fluid (CSF; 19 leukocytes/ $\mu$, protein $630 \mathrm{mg} / \mathrm{l}$, with no intrathecal synthesis of immunoglobulins (including isoelectric focusing)) suggested an inflammatory disease, but no infectious cause was found systemically or in CSF (i.e. using bacterial and fungal cultures, tests for toxoplasmosis and pneumocystis, and PCR for cytomegalovirus (CMV), herpes simplex virus, varicella-zoster virus, Epstein-Barr virus, human herpesvirus 6, polyomaviruses JCV and BKV, and enteroviruses). Assuming encephalitis as the only manifestation of GvHD, treatment with steroids and antiepileptics was started on day 246 . The cognitive deficits improved over the next few months and steroids were slowly reduced. On day 425 , her husband reported recurring mild personality changes, and 2 months later she had another seizure. Cerebral magnetic resonance imaging (MRI; fig. 1) showed confluent periventricular lesions without gadolinium enhancement or infratentorial lesion, and CSF was unchanged. Thus, IS with steroids was restarted complemented with MMF, resulting in improvement of neurological symptoms. In 2009, seizures recurred in form of transient aphasia/dysarthria during reduction of steroids. Despite progressive periventricular lesions in cerebral MRI, she improved clinically, and the steroids were tapered.

In March 2010, she complained blurred vision and floaters on the right eye (visual acuity 1.0/1.0). External eye and anterior segment findings were normal without corneal staining, but lacrimal gland function was reduced in the Schirmer test $(4 \mathrm{~mm})$. Both eyes showed whitish circumscribed retinal infiltrations in the mid-periphery of the fundus, cellular infiltration of the vitreous, and mild bilateral optic disc edema (figs. 2A and 2B). 2 years previously (i.e. 8 months after alloHSCT), the funduscopy had been normal. Goldmann perimetry showed regular visual fields despite a blind spot enlargement on the left eye. Visual evoked potentials revealed bilateral delayed latency (P100 right side $121 \mathrm{~ms}$, left side $124 \mathrm{~ms}$,
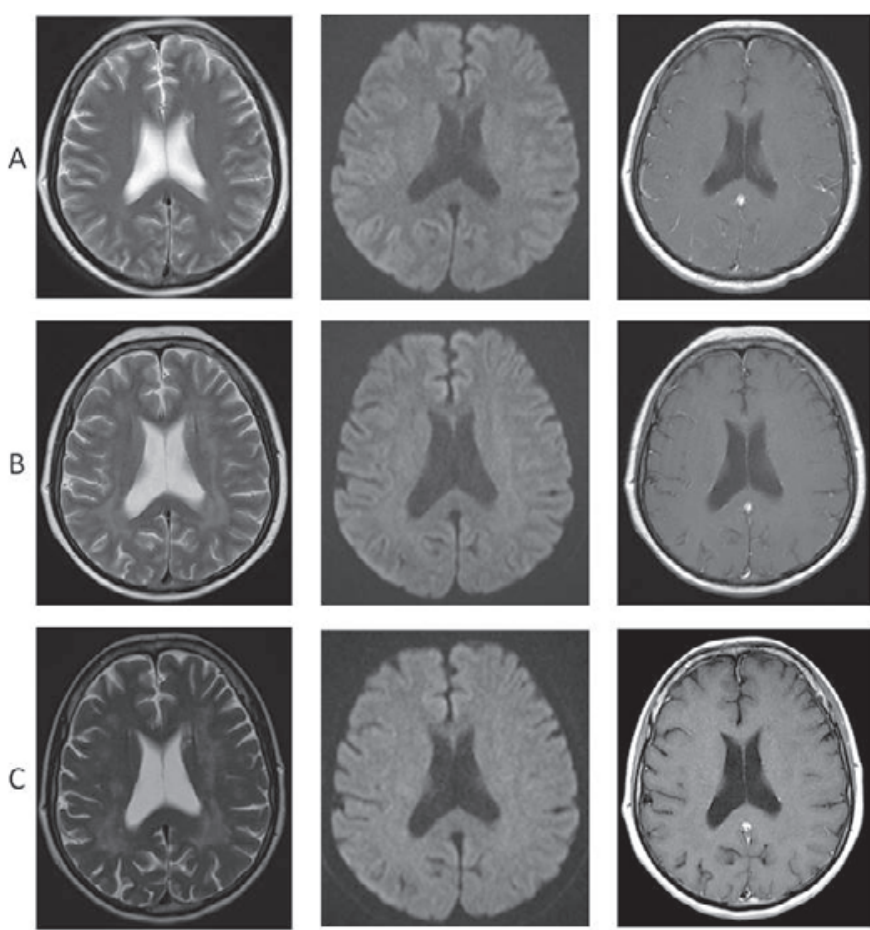

Fig. 1. Cerebral magnetic resonance imaging (MRI). (A) Day 162 after allogeneic peripheral blood stem cell transplantation (alloHSCT): without pathological findings (axial T2-weighted turbo spin echo (TSE), diffusion-weighted im aging (DWI), T1-weighted SE post contrast). (B) Day 430: confluent periventricular lesions without acute changes on DWI and no contrast enhancement (axial T2 TSE, DWI, T1 SE post contrast). (C) Day 1185: progressive confluating periventricular lesions without acute changes on DWI and no contrast enhancement (axial T2 TSE, DWI, T1 SE post contrast).

normal $<120 \mathrm{~ms}$ ); MRI did not suggest optic neuritis. The oncological and neurological work-up ruled out acute infectious diseases as CMV infection or re-activation, lymphoma or leukemic manifestations, and other GvHD manifestations. Symptoms and signs resolved under continued systemic IS treatment with prednisolone and MMF, leaving mildly pigmented retinal scars (fig. 2C). The patient developed multiple osteonecrotic lesions (femur and tibia) and, 4 years after transplantation, mild anemia and thrombocytopenia. Bone marrow (BM) biopsy showed mild marrow hypoplasia, but complete donor chimerism without signs for myelodysplasia and normal cytogenetics. However, staining for osteopontin and endothelial cells (CD34) revealed a decline in vascularization within the BM and a significant reduction of osteopontin expression. After subsequent tapering and termination of IS, at 52 months after alloHSCT, the patient developed cutaneous $\mathrm{CGvHD}$ for the first time with sclerotic features, which was treated with extracorporal photopheresis, resulting in resolution and normalization of blood counts. Currently, she is alive and doing well 94 months after transplantation.

\section{Discussion}

While cGvHD frequently involves epithelial structures and fascia, it rarely involves the central nervous system (CNS) [6-8]. Since no specific criteria for CNS manifestation of cGvHD have been established, the diagnosis was based on exclusion of other causes and response to immunosuppressive treatment $[6,8]$. In accordance with the consensus criteria for cerebral GvHD $[6,8]$, the likelihood of a CNS manifestation of cGvHD, as explanation of symptoms of our patient, is high since: (1) neurological symptoms, including 

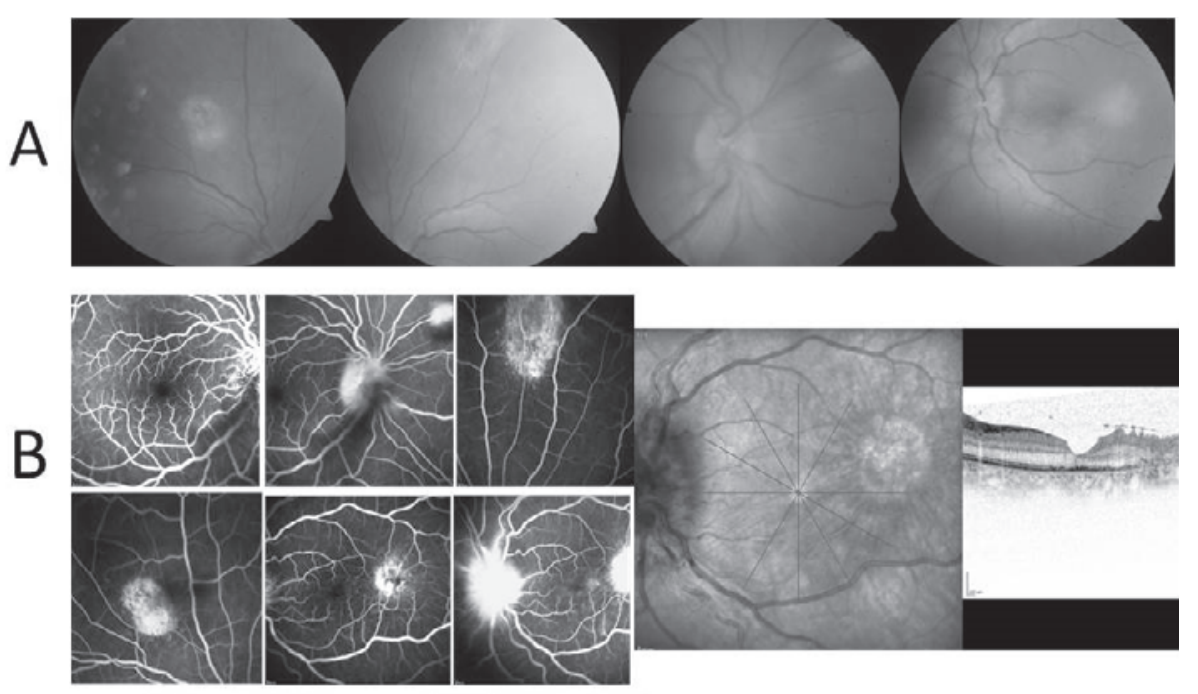

Fig. 2. (A) Whitish retinal and chorioretinal lesions in the mid-periphery of the right and left fundus, mild optic disc edema and mild cellular infiltration of the vitreous can be seen. (B) During fluorescein angiography, staining of retinal/chorioretinal lesions and leakage at the optic disc were observed. Optical coherence tomography (OCT) scan revealed (chorio-) retinal scarring with irregular retinal cell layers. (C) Whitish, mildly pigmented retinal/chorioretinal scars on the right and left fundus can be observed. OCT scan showed (chorio-) retinal scarring with disorganized retinal layers.

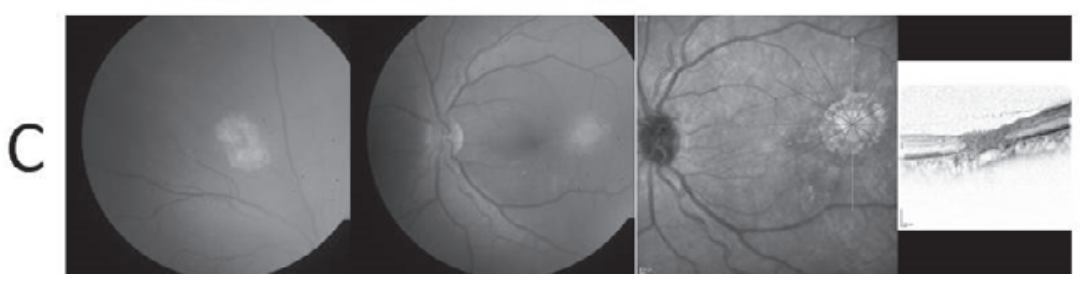

seizures, correlated closely with the intensity of IS; (2) withdrawal of steroids led to worsening of symptoms; and (3) other causes were excluded. To our knowledge this is the first time that a putative retinal involvement of cerebral manifestations of cGvHD, with retinal infiltrations documented in the absence of other causes, has been observed. This suggests that cGvHD may involve the retina directly and has to be differentiated from infectious diseases (e.g. CMV retinitis) and retinal microangiopathy (e.g. radiation retinopathy), which are frequently seen in patients after HSCT $[9,10]$. While the pathogenesis is unknown, the decline of vascularization observed in the BM and the retinal infiltration with subsequent scarring suggest a vasculitis-mediated process in our patient. In a murine GvHD model, a loss of osteoblasts and endothelial cells (explaining cytopenia and osteonecrosis) have been reported [11], both key components of the stem niche. Biedermann et al. [12] described $\mathrm{CD}^{+}$-mediated vasculitis in cutaneous $\mathrm{CGvHD}$, leading to a loss of microvessels. The lack of acute brain infarction in the MRIs might be similar to the situation in vascular dementia. As CNS, retina and bone structures are extremely sensitive to vascular damage (possibly aggravated by the use of steroids causing avascular bone necrosis), these systems primarily show a diffuse vascular process of microvessels. In addition, the present case indicates a potential risk for ocular posterior pole involvement in patients with cerebral manifestations of cGvHD. Based on the finding for our patient, cerebral involvement may occur as the only clinical manifestation of GvHD including retinal involvement, and funduscopy should be considered as a diagnostic tool.

\section{Disclosure Statement}

The authors declare no conflict of interest.

\section{References}

1 Kim SK, Smith JA, Dunn JP: Chronic ocular graft versus host disease; in: Vogelsang GB, Pavletic SZ (eds): Chronic Graft Versus Host Disease: Interdisciplinary Management. New York: Cambridge University Press, 2009, pp. 199-206.

2 Dietrich-Ntoukas T, Cursiefen C, Westekemper H, et al.: Diagnosis and treatment of ocular chronic graftversus-host disease: Report from the German-Austrian-Swiss Consensus Conference on Clinical Practice in chronic GVHD. Cornea 2012;31:299-310.

3 Ogawa Y, Kim SK, Dana R, et al.: International Chronic Ocular Graft-vs-Host-Disease (GVHD) Consensus Group: Proposed diagnostic criteria for chronic GVHD. Sci Rep 2013;3:3419.

4 Kim SK: Update on ocular graft versus host disease. Curr Opin Ophthalmol 2006;17:344-348.
Finke J, Bethge WA, Schmoor C, et al.: Standard graftversus-host disease prophylaxis with or without anti-Tcell globulin in haematopoietic cell transplantation from matched unrelated donors: A randomised, openlabel, multicentre phase 3 trial. Lancet Oncol 2009;10: 855-864.

6 Grauer O, Wolff D, Bertz H, et al.: Neurological manifestations of chronic graft-versus-host disease after allogeneic haematopoietic stem cell transplantation: Report from the Consensus Conference on Clinical Practice in chronic graft-versus-host disease. Brain 2010; 133:2852-2865.

7 Kamble RT, Chang CC, Sanchez S, Carrum G: Central nervous system graft-versus-host disease: Report of two cases and literature review. Bone Marrow Transplant 2007;39:49-52.
Openshaw H, Ressler JA, Snyder DS: Lumbar puncture and subdural hygroma and hematomas in hematopoietic cell transplant patients. Bone Marrow Transplant 2008;41:791-795.

9 Bernauer W, Gratwohl A, Keller A, et al.: Microvasculopathy in the ocular fundus after bone marrow transplantation. Ann Intern Med 1991;115:925-930.

10 Tabbara KF, Al-Ghamdi A, Al-Mohareb F, et al.: Ocular findings after allogeneic hematopoietic stem cell transplantation. Ophthalmology 2009;116:1624-1629.

11 Shono Y, Ueha S, Wang Y, et al.: Bone marrow graftversus-host disease: Early destruction of hematopoietic niche after MHC-mismatched hematopoietic stem cell transplantation. Blood 2010;115:5401-5411.

12 Biedermann BC, Sahner S, Gregor M, et al.: Endothelial injury mediated by cytotoxic $\mathrm{T}$ lymphocytes and loss of microvessels in chronic graft versus host disease. Lancet 2002;359:2078-2083. 